\title{
Efficacy of Toric intraocular lens implantation in eyes with high myopia: A prospective, case-controlled observational study
}

\author{
TAO GUO ${ }^{1,2^{*}}$, PENG GAO $^{3 *}$, LI FANG $^{1,2}$, LI GUO $^{1,2}$, YUCHEN FAN $^{1,2}$ and CHANGGEN LIU ${ }^{4}$ \\ ${ }^{1}$ Department of Ophthalmology, Shanghai Ninth People's Hospital, Shanghai JiaoTong University School of Medicine; \\ ${ }^{2}$ Shanghai Key Laboratory of Orbital Diseases and Ocular Oncology, Shanghai 200011; ${ }^{3}$ Department of Ophthalmology, \\ Shanghai Tenth People's Hospital, Tongji University School of Medicine, Shanghai 200072; ${ }^{4}$ Department of \\ Technical Services, Shanghai ShuJin Information Technology Co., Ltd., Shanghai 201399, P.R. China
}

Received October 19, 2017; Accepted February 16, 2018

DOI: $10.3892 / \mathrm{etm} .2018 .6102$

\begin{abstract}
The present prospective clinical observational study aimed to evaluate the efficacy of Toric intraocular lenses (IOL) to achieve rotational stability and astigmatism correction in eyes with high myopia. A total of 27 consecutive cataract patients (39 eyes) with pre-existing corneal astigmatism (1.5-3.5 D) were divided into two groups according to their refractive status: One group of 18 eyes with high myopia -(12.5-6.0 D) and another group consisting of 21 eyes with emmetropia or low myopia (-3.0-0.0 D). All eyes underwent cataract phacoemulsification surgeries by the same surgeon, with the implantation of an AcrySof ${ }^{\circledR}$ Toric IOL at the pre-designed degree. Uncorrected visual acuity, best corrected visual acuity and phoropter examination results were recorded at the 1st day, 1st week, 1st month and 3rd month after the surgery. By analyzing digital images from slim-lamp photography with a self-designed software, the rotational stability was observed. The contrast sensitivity was also measured. No significant difference in the baseline and post-operative residual astigmatism was identified between the two groups $(\mathrm{P}>0.05)$. In addition, no significant difference in the degree of rotation was observed between the two groups. All patients had significantly improved visual quality after the surgery $(\mathrm{P}<0.05)$. In conclusion, the present study observed an equal astigmatism-correction efficiency and rotational stability in
\end{abstract}

Correspondence to: Professor Tao Guo, Department of Ophthalmology, Shanghai Ninth People's Hospital, Shanghai JiaoTong University School of Medicine, 280 Mohe Road, Baoshan, Shanghai 200011, P.R. China

E-mail: guotao9@hotmail.com

*Contributed equally

Abbreviations: Toric IOL, Toric intraocular lens; UCVA, uncorrected visual acuity; BCVA, best corrected visual acuity; AL, axial length; logMAR, logarithm of the minimum angle of resolution

Key words: Toric intraocular lenses, myopia, cataract surgery, rotational stability, astigmatism correction, visual quality the two groups. A second auxiliary spot-penetrating incision, removal of visco-elastic substances and tight adherence of the IOL to the posterior capsular membrane are essential for a successful surgery.

\section{Introduction}

With the rapid development of cataract surgery, post-operative refractive errors have been markedly reduced (1). Compared to myopia or hyperopia, which may be easily corrected by spherical lenses, corneal astigmatism of $>1.5 \mathrm{D}$ occurs in $19 \%$ of cataract patients, and its correction with glasses remains less efficient. Traditional surgical methods include making incisions in the cornea, such as limbal or corneal relaxing incisions, the outcome of which has been proved somewhat unpredictable and variable as a result of different healing responses and operating skills. Other disadvantages include possible risks of overcorrection, perforation and wound leakage, which have all limited this application in clinical practice (2-4).

In comparison with the abovementioned methods, Toric intraocular lenses (Toric IOL) are able to more precisely correct astigmatism. Numerous studies have demonstrated the efficacy of Toric IOL vs. spherical IOL plus limbal relaxing incisions in correcting astigmatism $(5,6)$. According to a retrospective study performed by Sun et al (7), the mean post-operative refractive cylinder was smaller in the Toric IOL group compared with that in the group subjected to spherical IOL implantation combined with limbal relaxing incisions $(\mathrm{P}<0.05)$, suggesting its advantages in efficiency and precision.

However, the crucial factor for the obtainment of such positive results is the long-term maintenance of a concrete rotational stability of the Toric IOL in the capsular bag (8). A small deviation of 10 degrees is estimated to reduce the correction power by $35 \%$ (9). Capsular bag shrinkage due to fibrosis, which occurs in the first three months post-operatively, has been considered as the crucial recovery time (10).

Other risk factors affecting the rotational stability of Toric IOL include axial length (AL), IOL design and material, axis of IOL alignment, capsulorhexis size, capsular bag diameter and overall haptic diameter $(6,11)$. In a study by Shah et al (12), a higher rotation was observed in eyes with a greater AL, 
and it was indicated that AL is the primary factor affecting rotational stability, rendering a high AL a disadvantage. The above study reported a worse outcome for Toric IOL implantation in eyes with high myopia and a high AL. Further factors associated with a worse outcome of Toric IOL implantation in myopia eyes include looser capsular bag, high chamber depth and vitreous liquefaction. All of these factors increase the difficulty of performing the operation, and may possibly lead to a worse prognosis $(13,14)$.

The outcome of Toric IOL implantation in eyes with high myopia has remained to be quantitatively assessed and associated studies are currently lacking. With this regard, the present prospective observational study was performed to compare the efficiency of astigmatism correction by Toric IOL and the rotational stability achieved between eyes with high myopia and those with low myopia or emmetropia, and thus to evaluate the clinical application of Toric IOL in patients with astigmatism and high myopia.

\section{Patients and methods}

Patients. The present prospective clinical observational study included 27 cataract patients who received phacoemulsification surgery from January 2011 to June 2012 at the Department of Ophthalmology of the Tenth People's Hospital (Shanghai, China). The inclusion criteria were as follows: Pre-existing corneal regular astigmatism $>1.50 \mathrm{D}$ with conformable results from IOL master and corneal topography; at least one obvious episcleral vessel near the limbus for use as a reference to align the images acquired from the follow-ups; clear display and explicit identification of the axis-marking spots on the lens, without the mask of undilated pupils or capsular opacity as a result of post-operative proliferation. The exclusion criteria were as follows: Pre-operative abnormalities as irregular corneal astigmatism, zonular weakness, a history of glaucoma, uveitis, macular disease, intraocular or keratorefractive surgery and intraoperative complications including posterior capsule rupture. All patients provided written informed consent prior to the study according to the Declaration of Helsinki and the protocol of the study was approved by the Ethics Committee of Shanghai Tenth People's Hospital.

Pre-operative assessment. The age and gender of each patient were recorded. Pre-operative assessment included uncorrected visual acuity (UCVA), best-corrected visual acuity (BCVA), AL (IOL master; Carl Zeiss AG, Oberkochen, Germany); phoropter examination (Nidek 9000; Nidek Co., Ltd., Gamagori, Aichi, Japan) to determine the refractive status, corneal topography (Orbscan; Bausch \& Lomb, Bridgewater, NJ, USA) and optical coherence interferometry measurement (IOL master; Karl Zeiss AG) to determine the preexisting corneal astigmatism, and the contrast sensitivity (Vector Vision; Greenville, OH, USA) under BCVA to assess the visual quality at the photopic and scotopic status. Other pre-operative examinations for cataract surgery, including the corneal endothelial scope, were also routinely performed.

Groups and measurements. According to the pre-operative refractive status, all patients were divided into 2 groups: Group1, high myopia (-12.5-6.0 D) group including 11 patients
(18 eyes); and Group 2, emmetropia or low myopia group (-3.0-0.0 D) consisting of 16 patients (21 eyes). The eyes in each group received cataract phacoemulsification surgeries with the implantation of an AcrySof ${ }^{\circledR}$ Toric IOL (Alcon Laboratories, Inc., Fort Worth, TX, USA) under local anesthesia. The horizontal axis was marked pre-operatively at the limbus with a marker pen under the slit lamp (BQ900; Haag Streit, Köniz, Bern, Switzerland). While the alignment axis of the Toric lens was pre-generated with the Calculation Tool software (version 3.2.4) provided on the Alcon website (http://www.acrysoftoriccalculator.com) according to the data from the IOL master examination and marked with a marker ring at the beginning of the surgery. According to the different pre-operative refractive status and personal reading habits, the post-operative refractive status was set as emmetropia in group 2 to generate a good far visual acuity, while it was set at $-3.0 \mathrm{D}$ of myopia for patients in group 1 to achieve a clear near vision for their reading habits. These settings were fully accepted by the patients after it was explained to them prior to the surgery. Different models of Toric IOL from SA60T3 to SA60T8 were selected according to the diopter of corneal astigmatism calculated according to the manufacturer's instructions for the AcrySof ${ }^{\circledR}$ Toric IOL.

Surgical procedure. The standard surgical procedure for the cataract phacoemulsification, which was performed by the same surgeon (TG) for all cases, was as follows: A $3.0 \mathrm{~mm}$-wide superior-temporal clear limbal incision at 120 degrees, an auxiliary spot-penetrating incision at 30 degrees, continuous curvilinear capsulorhexis, and phacoemulsification with the cleaver technique and irrigation/aspiration. Thereafter, the Toric IOL was implanted into the capsular bag with a Monarch II injector and a B-Cartridge, and subsequently rotated clockwise to the target alignment axis as specified prior to surgery. In the standard procedure of the surgery, the posterior capsule was carefully polished as routine practice using an irrigation and aspiration handpiece tip, while the anterior capsule was not polished.

To ensure stable implantation of the IOL in the capsular bag and prevent its instant shift or rotation, several specific techniques were applied: First, in certain cases, a second auxiliary spot-penetrating incision at 180 degrees was adopted to facilitate the precise rotation of the IOL by the surgeons to the target alignment axis. This second auxiliary incision, which was made in the same way as the first one at 30 degrees, neither increased the complexity of the surgery nor added to the post-operative astigmatism. Furthermore, the visco-elastic substances at the inferior capsular membrane-IOL gap and the posterior capsular membrane-IOL gap were completely removed, as residual reagent may cause a rotation of the IOL in the capsular bag. Finally, after the accurate alignment of the IOL, the lens was pushed down at the optical center to tightly adhere to the posterior capsular membrane and the incisions were then made watertight, thus preventing an upward movement of the IOL caused by the surge of the anterior chamber.

Post-operative treatment and examination. Tobramicin and dexamethasone eye drops (Alcon Laboratories, Inc.) were applied 4 times daily for 2-3 weeks. The follow-up 
A

ه2 Comparison of Pre.op And Post-op Image Software Vz.0 Dept. of Ophthalmology. Shanghai Tenth People's Hospita
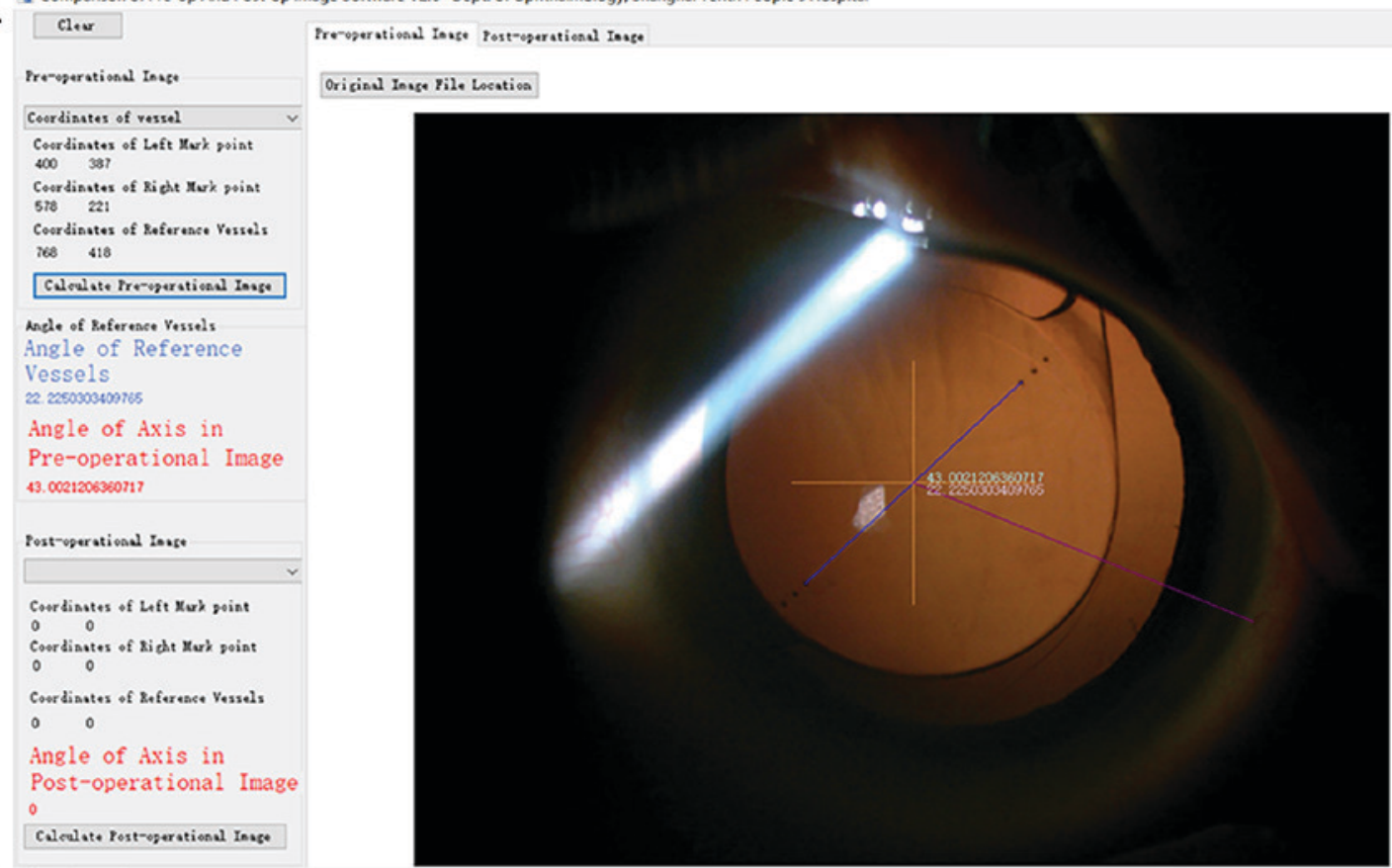

B

Cles

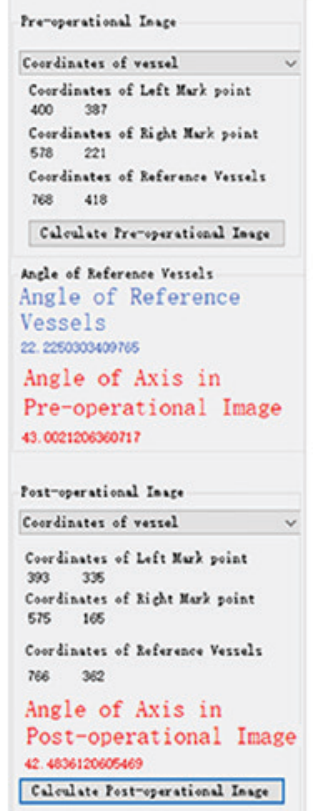

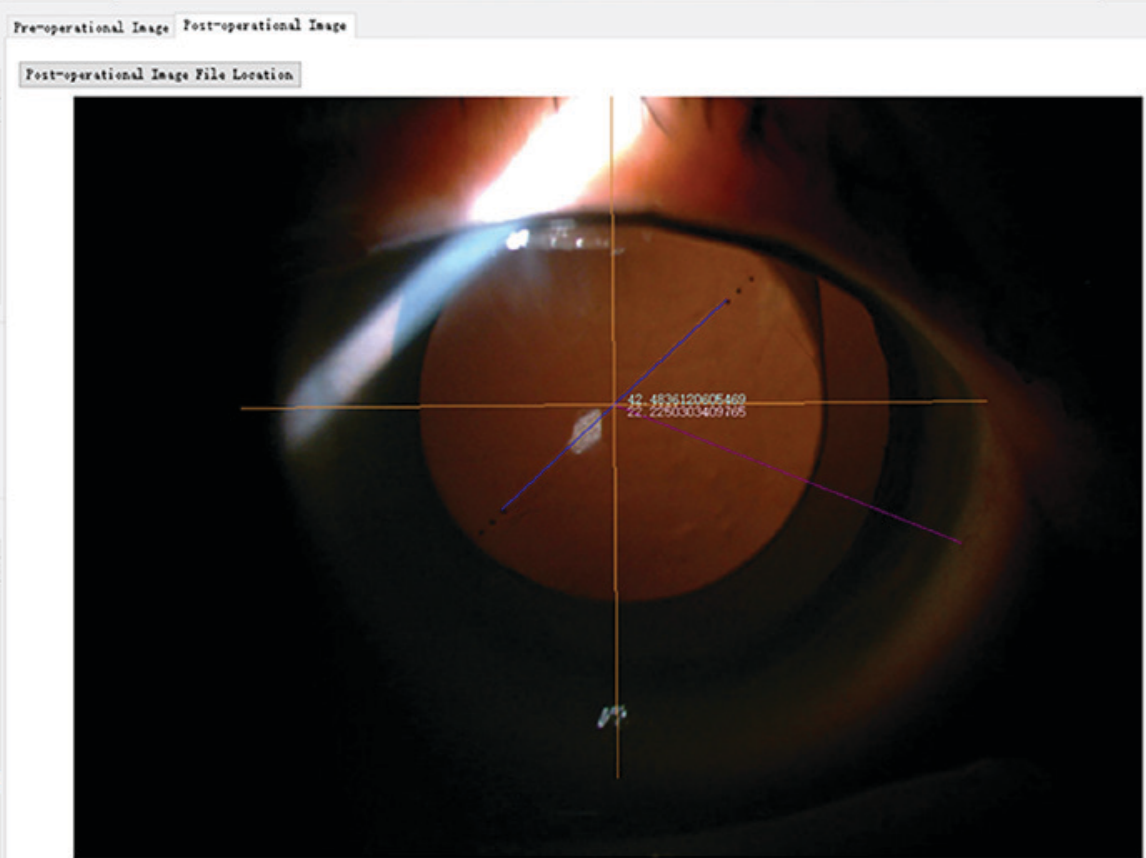

Figure 1. Image analysis for the calculation of rotational stability of Toric IOLs with purpose-designed software. (A) Post-1 day and (B) post-1 week image analyzed with the calculation software (magnification, x16). By specifying two symmetrical marking spots on the Toric IOL plates, the coordinate axis was placed. One same point on the characterized episcleral vessel, which was always distinctly visible among all of the post-operative images, was selected as the anchor point. By keeping the anchor-point angle unchanged within the coordinate axis, the post-operative image was placed correspondingly, and the rotation angle of the IOL was calculated accordingly and generated automatically. IOL, intraocular lenses.

was arranged at the 1st day, 1st week, 1st and 3rd month post-operatively. The following tests were performed: UCVA, BCVA, phoropter examination and contrast sensitivity. The BCVA was converted to the logarithm of the minimum angle of resolution $(\log M A R)$. The abilities to count fingers, see hand motion and perceive light were assigned the $\log \mathrm{MAR}$ units of 1.9, 2.3 and 2.7, respectively.

To examine the rotational stability of the IOL in the capsular bag, images of the anterior segment were captured and then analyzed by using purpose-designed comparison of Pre-op And Post-op Image Software V2.0 (supplied by the Department of Ophthalmology, Shanghai Tenth People's Hospital and Shanghai ShuJin Information Technology Co., Ltd., Shanghai, China), which was able to align the images from different time-points according to the reference episcleral vessel, as indicated in Fig. 1. The axis shift of the IOL was then determined using the standard astigmatism chart.

Statistical analysis. Statistical data analysis was performed with SPSS 10.0 software (SPSS, Inc., Chicago, IL, USA). Values are expressed as the mean \pm standard deviation. For comparative statistics, the Wilcoxon signed-rank test, chi-square test 
Table I. Patient demographics and pre-operative data.

\begin{tabular}{lccr}
\hline Parameter & High myopia (group 1; n=11) & Emmetropia or low myopia (group 2; n=16) & P-value \\
\hline Eyes & 18 & 21 & \\
Sex (\%) & & & \\
Male & $6(54.55)$ & $7(43.75)$ & 0.704 \\
Female & $5(45.45)$ & $9(56.25)$ & 0.704 \\
Age (years) & $66.5 \pm 7.5 ; 67(57-76)$ & $69.3 \pm 8.2 ; 69(61-75)$ & 0.373 \\
Mean spherical equivalent (D) & $-9.5 \pm 2.7 ;-9.75-(12.50-6.00)$ & $-2.25 \pm 1.1 ;-1.75(-3.75-0.00)$ & $<0.001$ \\
AL (mm) & $26.43 \pm 0.82 ; 26.88(25.27-27.63)$ & $24.16 \pm 0.68 ; 24.06(23.36-25.02)$ & $<0.001$ \\
IOL master diopter (D) & $6.37 \pm 3.68$ & $20.42 \pm 2.30$ & $<0.001$ \\
Corneal astigmatism & & & \\
IOL master cylinder (D) & $2.23 \pm 0.63$ & $2.07 \pm 0.58$ & 0.414 \\
Orbscan topography (D) & $2.17 \pm 0.59$ & $2.11 \pm 0.61$ & 0.758 \\
Phoropter cylinder (D) & $1.89 \pm 0.61$ & $1.93 \pm 0.77$ & 0.860
\end{tabular}

${ }^{a}$ Values are expressed as the mean \pm standard deviation; median (range). IOL, intraocular lenses; AL, axial length.

and one way ANOVA with an SNK post hoc test were used for independent samples. $\mathrm{P}<0.05$ was considered to indicate a statistically significant difference.

\section{Results}

Patient characteristics. The demographics of the cohort and pre-operative data are presented in Table I. No significant differences in pre-operative corneal astigmatism were noted. However, the mean spherical equivalent and AL were significantly different between the two groups $(\mathrm{P}<0.05)$.

Improvement of UCVA, BCVA and mean spherical equivalent after surgery. The visual acuity was reduced in the UCVA group compared with the BCVA group at the pre-operative stage (Table II). At the 1st day post-operative the UCVA and BCVA groups were significantly improved compared with the pre-operative stage $(\mathrm{P}<0.001)$. At all stages, pre- and post-operative UCVA and BCVA were better in group 2 (low myopia and emmetropia; $\mathrm{P}<0.05$ ) (Table II). The post-operative mean spherical equivalent was similar to the preset refractive endpoint (Table II). In group 1 the post-operative mean spherical equivalent was changed to around $-3.0 \mathrm{D}$ as designed, while in group 2 it switched from low myopia or emmetropia pre-operatively to near emmetropia post-operatively as designed (Table II).

Improvement of astigmatism after surgery. In each of the two groups, the astigmatism was significantly reduced after surgery $(\mathrm{P}<0.05)$. No significant difference in post-operative residual astigmatism between the two groups was noted ( $\mathrm{P}>0.05$; Fig. 2).

Toric IOL rotation in the two groups. The Toric IOL rotation in groups 1 and 2, respectively, was quantified as $3.91 \pm 1.73$ vs. $3.89 \pm 1.62$ degrees at 1 day, $3.97 \pm 1.55$ vs. $3.93 \pm 1.41$ degrees at 1 week, $4.25 \pm 1.42$ vs. $4.11 \pm 1.57$ degrees at 1 month and $4.27 \pm 1.51$ vs. $4.16 \pm 1.55$ degrees at 3 months post-surgery. Although the curves of the rotational degree exhibit a slight shift from 1 week to 1 month post-surgery in each group, there was no significant difference in the degree of rotation between the two groups during the entire post-operative period (P>0.05; Figs. 1 and 3).

Toric IOL achieves better photopic and scotopic contrast sensitivity in patients with astigmatism and high myopia. Photopic and scotopic contrast sensitivity was significantly improved at the third post-operative month when compared with the baseline status $(\mathrm{P}<0.05)$. Group 1 achieved better photopic and scotopic contrast sensitivity than group 2 ( $\mathrm{P}<0.05$; Fig. 4).

\section{Discussion}

The development and implementation of the Toric IOL provided surgeons with an alternative to correct astigmatism during phacoemulsification cataract surgery. Emerging studies have indicated high accuracy and efficacy of Toric IOL in correcting astigmatism compared to spherical IOLs plus limbal relaxing incisions $(5,6)$. In the present study, comparable results regarding the efficiency of astigmatism correction and rotational stability were obtained. The two groups exhibited improvements in the UCVA and BCVA, and reduced astigmatism. No significant difference in residual astigmatism was observed between the two groups. During the follow-up, no significant difference in the rotation degree was noted between the two groups $(\mathrm{P}>0.05)$. The present results corroborate with those of previous studies $(7,11,12)$. Therefore, it may be concluded that implantation of Toric IOL in high myopia eyes is effective.

A high AL, a significantly looser capsular bag, higher chamber depth and vitreous liquefaction in eyes with high myopia may increase the difficulty of the operation as follows (13-15): The higher chamber depth in the surgery increases the vertical distance for operation and calls for a higher depth of the visual field, along with leakage from the corneal incision, and thus, surgeons are required to apply their 
Table II. Visual acuity and mean spherical equivalent at the pre-operative stage and at post-operative follow-up.

\begin{tabular}{|c|c|c|c|}
\hline Parameter & High myopia (group $1 ; \mathrm{n}=11$ ) & Emmetropia or low myopia (group $2 ; n=16$ ) & P-value \\
\hline \multicolumn{4}{|c|}{ Pre-operative visual acuity } \\
\hline UCVA & $0.04 \pm 0.08$ & $0.23 \pm 0.11$ & $<0.001$ \\
\hline BCVA & $0.13 \pm 0.11$ & $0.28 \pm 0.12$ & $<0.001$ \\
\hline \multicolumn{4}{|c|}{ Post-operative visual acuity } \\
\hline \multicolumn{4}{|l|}{ UCVA } \\
\hline 1st day & $0.21 \pm 0.18$ & $0.67 \pm 0.11$ & $<0.001$ \\
\hline 1st week & $0.26 \pm 0.16$ & $0.69 \pm 0.21$ & $<0.001$ \\
\hline 1st month & $0.27 \pm 0.17$ & $0.77 \pm 0.20$ & $<0.001$ \\
\hline 3rd month & $0.28 \pm 0.18$ & $0.72 \pm 0.19$ & $<0.001$ \\
\hline \multicolumn{4}{|l|}{ BCVA } \\
\hline 1st day & $0.49 \pm 0.21$ & $0.69 \pm 0.13$ & $<0.001$ \\
\hline 1st week & $0.57 \pm 0.23$ & $0.70 \pm 0.15$ & 0.041 \\
\hline 1st month & $0.58 \pm 0.24$ & $0.72 \pm 0.16$ & 0.036 \\
\hline 3rd month & $0.60 \pm 0.22$ & $0.73 \pm 0.17$ & 0.045 \\
\hline \multicolumn{4}{|c|}{ Mean spherical equivalent (D) } \\
\hline Pre-operative & $-9.5 \pm 2.7$ & $-2.25 \pm 1.1$ & $<0.001$ \\
\hline 1st day & $-2.65 \pm 0.41$ & $-0.53 \pm 0.32$ & $<0.001$ \\
\hline 1st week & $-2.77 \pm 0.38$ & $-0.29 \pm 0.36$ & $<0.001$ \\
\hline 1 st month & $-2.83 \pm 0.37$ & $-0.26 \pm 0.31$ & $<0.001$ \\
\hline 3rd month & $-2.79 \pm 0.46$ & $-0.28 \pm 0.29$ & $<0.001$ \\
\hline
\end{tabular}

UCVA, uncorrected visual acuity; BCVA, best corrected visual acuity.

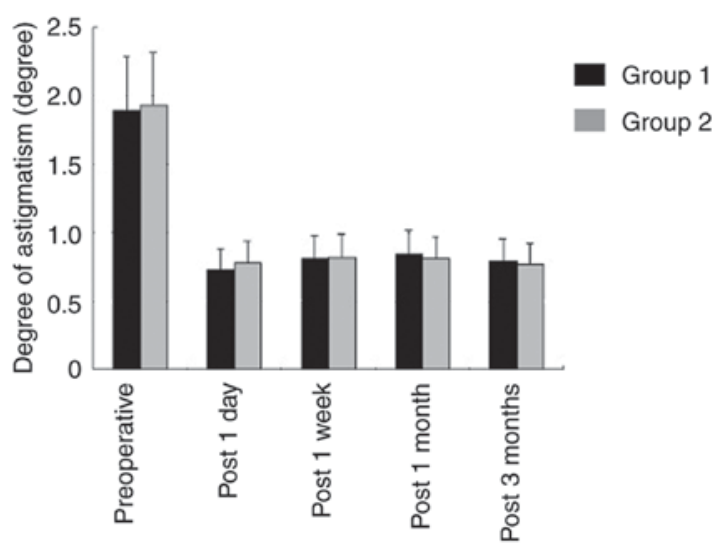

Figure 2. Refractive cylinder degree at the pre- and post-operative stage. The degree of post-operative astigmatism was significantly less than the pre-operative degree $(\mathrm{P}<0.05)$, while there was no significant difference in the post-operative residual astigmatism between the two groups during the entire follow-up $(\mathrm{P}>0.05)$.

instruments in a more vertical angle under compromised visualization. A looser capsular bag allows for more room for the IOL to move unpredictably and induces instability. A relaxed zonular ligament causes higher difficulty in IOL restoration. These issues may possibly lead to a worse outcome of Toric IOL implantation in high-myopia eyes, thus limiting its application.

Specific surgical techniques are essential in performing the surgery. With the help of the second auxiliary spot-penetrating incision at 180 degrees, IOLs are easier to rotate. Complete

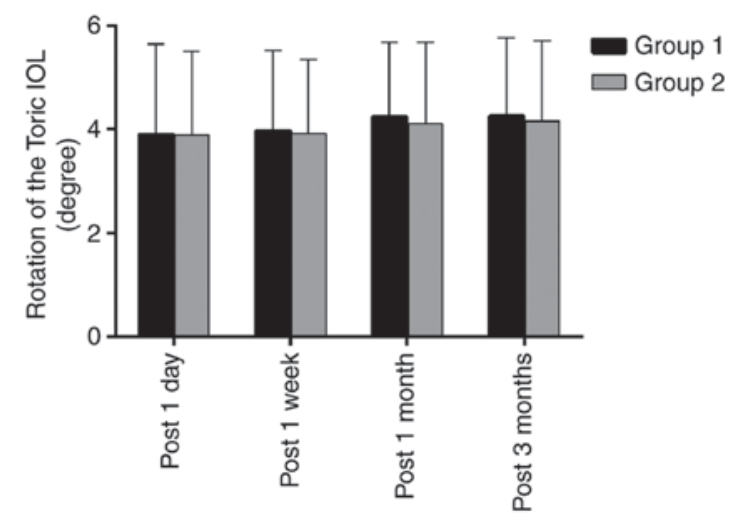

Figure 3. Rotational stability of Toric IOL. IOL, intraocular lenses.

removal of visco-elastic substances ensured stable IOL implantation in the capsular bag. Irrigation and aspiration provided a tight adherence of the IOL to the posterior capsular membrane. All of these procedures contribute to maintain the stability of Toric IOLs.

As for the pre- and post-operative UCVA and BCVA, group 2 (emmetropia or low-myopia eyes) had better vision group 1 (high-myopia eyes). A similar trend was observed for pre- and post-operative contrast sensitivity under the photopic and scotopic status. This result may be explained by the larger optical aberrations in the high-myopia eyes at low-order (e.g., myopia and astigmatism) and high-order (e.g., coma aberration) levels (13). However, each of the two 

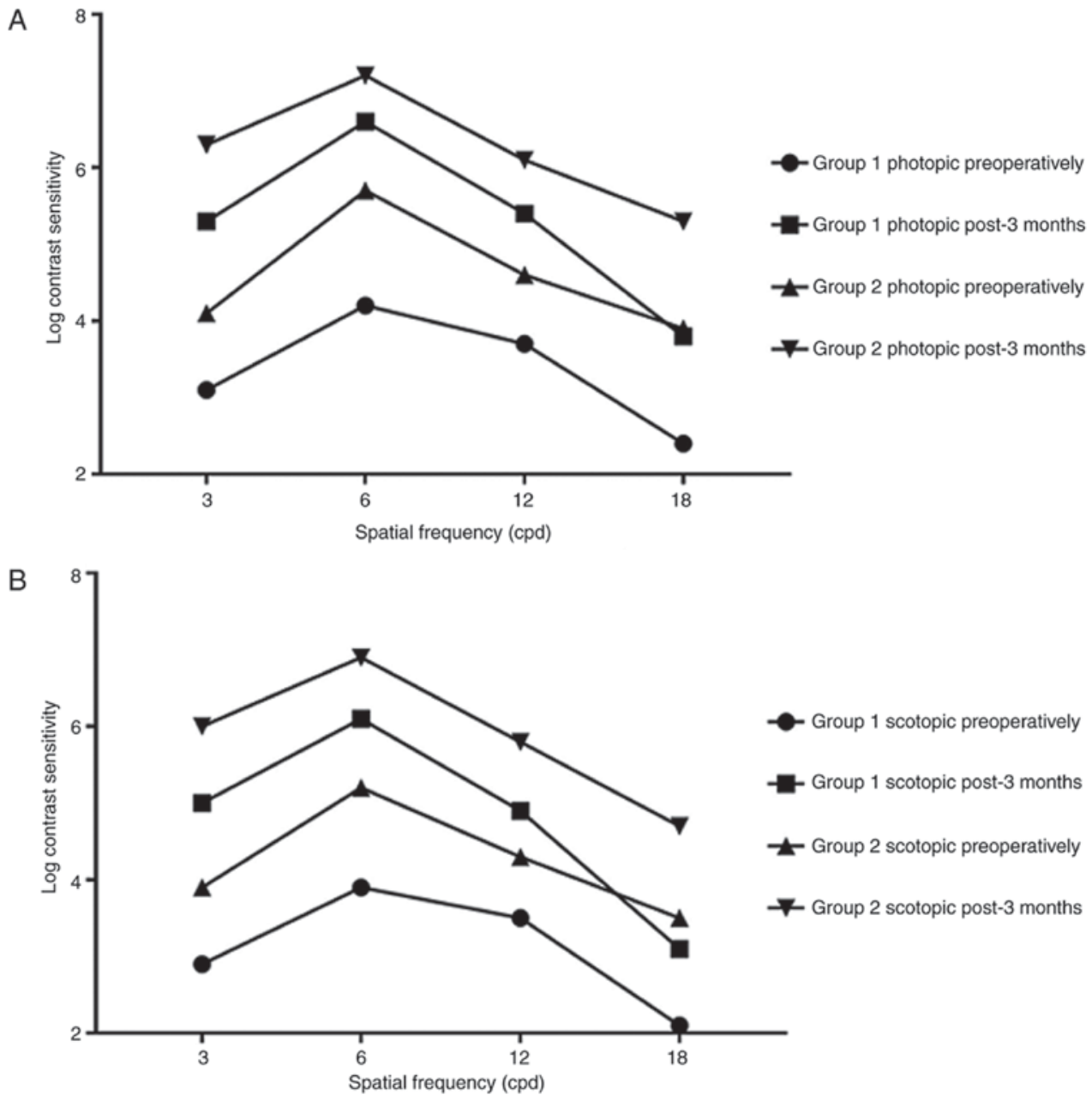

Figure 4. Contrast sensitivity under photopic and scotopic conditions of the two groups. (A) Photopic status and (B) scotopic status. The post-operative contrast sensitivity under photopic and scotopic conditions was significantly improved at the 3rd month post-operatively compared with that in each respective group pre-operatively $(\mathrm{P}<0.05)$. Under the two conditions, group 1 remained lower than group 2 during the entire period $(\mathrm{P}<0.05)$.

groups gained a significant improvement in UCVA, BCVA and contrast sensitivity. For far vision, patients only required myopia-correcting glasses post-operatively rather than myopia-astigmatism-correcting glasses used at the pre-operative stage, and for near vision, no glasses were required rather than cylinder glasses used at the pre-operative stage. However, according to several previous studies, controversy remains regarding this issue (16-19). Further investigation and discussion is therefore required to validate the present findings.

Fibrosis has been considered the underlying cause for capsular shrinkage and IOL movement at the post-operative stage (10). In the present study, the curve of the rotational degree displays a slight shift from 1 week to 1 month post-operatively for both each of the two groups $(\mathrm{P}<0.05)$. This result conformed to those of previous studies, which revealed a climax of capsular bag shrinkage and in situ fibrosis at that time $(6,12)$. Previous studies also indicated that a high AL is a risk factor for Toric IOL rotation in high-myopia eyes; in addition, a thick lens, large capsular sac and relatively loose capsule are associated with the likelihood and amplitude of rotation $(12,20)$. Due to the above reasons, certain experts do not recommend the implantation of Toric IOL to correct astigmatism in cataract patients with high myopia (12), which conflicts with the present results. The discrepancy in results may arise from differences in the study sample and the period of observation. Differences in surgical procedures between various studies may also contribute to this. Further study with a larger sample size and long follow-up may clarify this. Furthermore, a previous study indicated that a higher anterior capsular opacification grade may decrease Toric IOL rotation, indicating that reducing the polishing of the anterior capsule may improve the rotational stability of a Toric IOL (20), a point that has not been addressed in the present study. In the present study the anterior capsule was no polished, which may have contributed to the rotational stability.

Various methods have been used to calculate the rotations of toric IOL. Becker et al (21) introduced digital photography recording through the slit lamp and operating microscope, and this method has become a standard for evaluating the centration and axial positioning of a Toric IOL (22). Viestenz and Langenbucher (23) then used the method of simultaneous slide projection to evaluate the rotation of the Toric lens. In the present study, images of the anterior segment were captured with digital cameras affiliated to the slit-lamp under dilated pupils, and the image was then analyzed with specific software, which was able to align the images from different time-points according to the reference of the episcleral vessel and then calculate the rotation of the IOL axis. This method 
was similar to that of Shah et al (12) and offered precise data for comparison. However, the fibrosis at the peripheral capsular bag possibly masked the marking spots in certain cases at the 1-month follow-up, which forced these cases to be excluded from the present study, leading to a loss of the sample.

To the best of our knowledge, the present study was the first to compare the visual outcomes of patients with varying degrees of myopia after Toric IOL implantation. An equal efficiency in astigmatism correction and rotational stability were achieved for groups 1 and 2, indicating a prospective future for the application of Toric IOLs in high-myopia eyes. However, the small size of the sample remains a limitation of the present study. Therefore, studies with a larger sample size as well as a longer follow-up are required to further investigate the rotational stability and visual quality of Toric IOL in high-myopia eyes with astigmatism.

\section{Acknowledgements}

Not applicable.

\section{Funding}

This work was supported by the Fund of Shanghai Ninth People's Hospital (grant no. syzrc2014-003) and The Science and Technology Commission of Shanghai (grant nos. 15411970000 and 17DZ2260100).

\section{Availability of data and materials}

The analyzed data sets generated during the study are available from the corresponding author on reasonable request.

\section{Authors' contributions}

TG and PG together designed the protocol, analyzed the data and drafted the manuscript. TG was also the surgeon who performed all the surgeries in the study. PG and CL designed the software for the calculation of the IOL rotation degree. LF, LG and YF contributed to data acquisition and examination of the patients. All authors agree with the submission and have full responsibility for all primary data. No part of this paper has been published or submitted elsewhere.

\section{Ethical approval and consent to participate}

All patients provided written informed consent prior to the study according to the Declaration of Helsinki and the study was approved by the Ethics Committee of Shanghai Tenth People's Hospital.

\section{Consent for publication}

Written informed consent was obtained from all patients for the publication of their data.

\section{Competing interests}

The authors declare that they have no competing interests.

\section{References}

1. Talley-Rostov A: Patient-centered care and refractive cataract surgery. Curr Opin Ophthalmol 19: 5-9, 2008.

2. Yang LH and Tang X: The research progress in treating astigmatism at the time of cataract surgery. Zhonghua Yan Ke Za Zhi 47: 573-576, 2011 (In Chinese).

3. Amesbury EC and Miller KM: Correction of astigmatism at the time of cataract surgery. Curr Opin Ophthalmol 20: 19-24, 2009.

4. Buckhurst PJ, Wolffsohn JS, Davies LN and Naroo SA: Surgical correction of astigmatism during cataract surgery. Clin Exp Optomet 93: 409-418, 2010.

5. Dick HB and Buchner SE: Toric phakic intraocular lenses. Ophthalmologe 104: 1032-1040, 2007 (In German).

6. Edrington TB: A literature review: The impact of rotational stabilization methods on toric soft contact lens performance. Cont Lens Anterior Eye 34: 104-110, 2011.

7. Sun XY, Vicary D, Montgomery P and Griffiths M: Toric intraocular lenses for correcting astigmatism in 130 eyes. Ophthalmology 107: 1776-1782, 2000.

8. Horn JD: Status of toric intraocular lenses. Curr Opin Ophthalmol 18: 58-61, 2007.

9. Viestenz A, Walter S, Viestenz A, Behrens-Baumann W and Langenbucher A: Toric intraocular lenses and correction of astigmatism. Ophthalmologe 104: 620-627, 2007 (In German).

10. Kurz S, Krummenauer F, Hacker P, Pfeiffer N and Dick HB: Capsular bag shrinkage after implantation of a capsular bending or capsular tension ring. J Cataract Refract Surg 31: 1915-1920, 2005.

11. Chang DF: Early rotational stability of the longer Staar toric intraocular lens: Fifty consecutive cases. J Cataract Refract Surg 29: 935-940, 2003.

12. Shah GD, Praveen MR, Vasavada AR, Vasavada VA, Rampal G and Shastry LR: Rotational stability of a toric intraocular lens: Influence of axial length and alignment in the capsular bag. J Cataract Refract Surg 38: 54-59, 2012.

13. Saw SM, Gazzard G, Shih-Yen EC and Chua WH: Myopia and associated pathological complications. Ophthalmic Physiol Opt 25: 381-391, 2005.

14. Liu YC, Xia WT, Zhou XT, Liu RJ, Bian SZ, Ying CL and Zhu GY: Eyeball structure changes in high myopic patients and their significance for forensic assessment. Fa Yi Xue Za Zhi 24: 356-360, 2008 (In Chinese).

15. Seward H, Packard R and Allen D: Management of cataract surgery in a high myope. Br J Ophthalmol 85: 1372-1378, 2001.

16. Hayashi K, Kondo H, Yoshida M, Manabe S and Hirata A: Higher-order aberrations and visual function in pseudophakic eyes with a toric intraocular lens. J Cataract Refract Surg 38: 1156-1165, 2012.

17. Kamiya K, Shimizu K, Aizawa D, Igarashi A, Komatsu M and Nakamura A: One-year follow-up of posterior chamber toric phakic intraocular lens implantation for moderate to high myopic astigmatism. Ophthalmology 117: 2287-2294, 2010.

18. Alió JL, Piñero DP, Tomás J and Plaza AB: Vector analysis of astigmatic changes after cataract surgery with implantation of a new toric multifocal intraocular lens. J Cataract Refract Surg 37: 1217-1229, 2011.

19. Visser N, Nuijts RM, de Vries NE and Bauer NJ: Visual outcomes and patient satisfaction after cataract surgery with toric multifocal intraocular lens implantation. J Cataract Refract Surg 37: 2034-2042, 2011.

20. Zhu X, He W, Zhang K and Lu Y: Factors influencing 1-year rotational stability of AcrySof Toric intraocular lenses. Br J Ophthalmol 100: 263-268, 2016.

21. Becker KA, Auffarth GU and Völcker HE: Measurement method for the determination of rotation and decentration of intraocular lenses. Ophthalmologe 101: 600-603, 2004 (In German).

22. Warlo I, Krummenauer F and Dick HB: Rotational stability in intraocular lenses with C-loop haptics versus $\mathrm{Z}$ haptics in cataract surgery. A prospective randomised comparison. Ophthalmologe 102: 987-992, 2005 (In German).

23. Viestenz A and Langenbucher A: Rotational stability of the eye in standard photography. Klin Monbl Augenheilkd 221: 262-265, 2004 (In German). 